\title{
Responsabilité et biotechnologie
}

Philippe Cullet

\section{OpenEdition \\ Journals}

Édition électronique

URL : http://journals.openedition.org/ress/478

DOI : $10.4000 /$ ress.478

ISSN : 1663-4446

\section{Éditeur}

Librairie Droz

\section{Édition imprimée}

Date de publication : 1 novembre 2004

Pagination : 181-199

ISBN : 2-600-00980-9

ISSN : 0048-8046

Référence électronique

Philippe Cullet, «Responsabilité et biotechnologie», Revue européenne des sciences sociales [En ligne], XLII-130 | 2004, mis en ligne le 13 novembre 2009, consulté le 19 avril 2019. URL : http:// journals.openedition.org/ress/478; DOI : 10.4000/ress.478 


\section{Philippe CULLET*}

\section{RESPONSABILITÉ ET BIOTECHNOLOGIE}

\section{INTRODUCTION}

L'introduction légale ou illégale d'organismes transgéniques dans l'environnement pose un certain nombre de questions juridiques concernant les conséquences environnementales et socio-économiques du génie génétique. Les instruments juridiques internationaux concernant la biotechnologie se préoccupent à présent principalement de questions concernant l'acceptation des organismes transgéniques, par exemple, à travers la mise en œuvre d'une approche de précaution. Il est possible de résoudre un certain nombre de questions fondamentales grâce à l'application de l'approche de précaution. Cependant, l'introduction directe ou indirecte, légale ou illégale, d'organismes transgéniques dans l'environnement est aujourd'hui un fait dans un certain nombre de pays. Etant donné qu'une approche de précaution ne peut que contribuer de façon marginale à prévenir les possibles conséquences néfastes faisant suite à l'introduction d'organismes transgéniques dans l'environnement, il convient d'explorer la question de la responsabilité civile et étatique. De fait, la question de la responsabilité des acteurs impliqués directement ou indirectement dans l'introduction d'organismes transgéniques dans l'environnement présente un certain nombre de problèmes spécifiques qui méritent d'être examinés en détail. Ceci est dû au fait que la question de la responsabilité dans le contexte de la biotechnologie nécessite le développement de règles tenant compte des spécificités des problèmes potentiels. Par ailleurs, la question de la responsabilité est une question importante dans le contexte du Protocole de Cartagena étant donné que les Etats membres devraient développer au cours des années à venir des règles spécifiques sur la responsabilité ${ }^{1}$.

Ce chapitre examine le développement de règles concernant la responsabilité des différents acteurs impliqués dans la dissémination d'organismes transgéniques dans l'environnement. La première section examine le cadre juridique existant en droit international pour attribuer la responsabilité en cas de dommage à l'environnement qui constitue le point de départ pour le développement de

* L'auteur tient à remercier tous les participants de l'Atelier sur la responsabilité dans le cadre du Protocole sur la biosécurité, Mombasa, Kenya, 22-26 septembre 2003 pour des débats théoriques et pratiques très stimulants ainsi que les participants du groupe interdisciplinaire du RIBios pour leur commentaires et suggestions sur une version préliminaire de ce chapitre.

1 Article 27 du Protocole de Cartagena sur la prévention des risques biotechnologiques, Montréal, 20 janv. 2000. 
règles sur la responsabilité dans le contexte de la biotechnologie. La deuxième section introduit les trois catégories principales de questions qui se posent au niveau de la responsabilité dans le domaine de la biotechnologie. Elle examine la question de la responsabilité pour dommage environnemental, pour dommage socio-économique et la question particulière du dommage réclamé par les détenteurs de brevets sur des semences transgéniques. Enfin, la dernière section propose quelques pistes concernant les options ouvertes aux Etats membres du Protocole de Cartagena concernant le développement de règles de responsabilité et les options ouvertes aux pays du Sud pour le développement de règles de responsabilité au niveau national.

\section{LA RESPONSABILITÉ POUR DOMMAGE À L'ENVIRONNEMENT}

La responsabilité constitue un instrument juridique permettant de prendre en compte les conséquences négatives d'une activité légale ou illégale en forçant l'auteur à assumer les conséquences financières ou autres des dommages causés. La responsabilité a différentes fonctions qui sont liées. Son objectif de base est d'offrir une forme de réparation qui permet l'indemnisation des victimes d'un dommage causé. Elle a également une fonction préventive en ce sens qu'elle offre aux promoteurs d'une activité à risque un instrument leur permettant d'orienter leur comportement en fonction des conséquences prévues en cas de dommages causés par l'activité en question. En d'autres termes la responsabilité peut promouvoir l'intériorisation des coûts environnementaux et sociaux qui constitue la base du principe du pollueur payeur. De plus, la responsabilité peut également être vue comme une forme d'incitation pour la mise en oeuvre des obligations environnementales en vigueur. En ce sens, elle contribue à imposer une approche de prévention du dommage aux acteurs entreprenant une activité dangereuse pour l'environnement. La responsabilité est donc liée de façon directe et indirecte à la prévention du dommage environnemental, un aspect fondamental du droit de l'environnement ${ }^{2}$.

La responsabilité a traditionnellement été conçue comme un instrument permettant l'indemnisation de dommages spécifiques subi par la partie lésée. Les parties en question peuvent être des individus, des entreprises ou des Etats dans les situations où les dommages sont transfrontières. Différents types de responsabilité existent suivant les situations spécifiques. Au niveau des relations entre Etats, le droit international a développé des règles qui prévoient que les Etats sont responsables de tout manquement à leurs obligations juridiques envers d'autres Etats. Au niveau des relations entre parties privées, on peut distinguer les dommages causés hors de toute relation contractuelle et les conséquences d'un acte ou d'une omission liés à une obligation contractuelle. Dans le premier cas, le droit anglo-saxon des torts a, par exemple, permis la résolution d'un grand nombre de situations, en particulier en ce qui concerne les plaintes entre propriétaires terriens pour diverses formes d'intrusion dans leurs droits de propriété. Les

\footnotetext{
Par ex. Cour international de justice, Projet Gabcíkovo-Nagymaros (Hongrie/Slovaquie), Arrêt
} du 25 septembre 1997, Cour internationale de justice-Recueil 1997, p. 7. 
torts sont particulièrement bien adaptés pour les plaintes concernant une atteinte aux droits économiques liés à la propriété. Cela peut inclure des dommages environnementaux qui ont des conséquences négatives du point de vue du propriétaire, y compris dans les cas où l'intrusion n'est pas visible à l'œil nu . Cependant, dans les cas de dommages à l'environnement, le droit des torts est d'une utilité limitée puisqu'il est centré sur les dommages aux personnes et à la propriété, ce qui rend difficile la considération de dommages purement environnementaux. Par ailleurs, le droit des torts est basé sur une approche bilatérale qui privilégie la considération de dommages individuels. L'indemnisation des dommages de masse subis par un nombre indéterminé d'individus qui sont fréquents dans le domaine de l'environnement ne se prête donc pas à une résolution à travers les instruments traditionnels du droit des torts.

Dans le cas d'une responsabilité contractuelle, l'obligation d'indemnisation est liée à la loi ou au traité qui défini les conditions qui déclenchent la responsabilité. Il est donc plus facilement envisageable dans ce contexte de prévoir une responsabilité pour dommages à l'environnement. Différents catégories de responsabilité ont été développées pour faire face à différents types de situations. La responsabilité peut tout d'abord être engagée si l'auteur a commis une faute, par exemple en ne respectant pas un devoir spécifique imposé par la loi soit intentionnellement soit par négligence. Dans le cas de cette responsabilité pour faute, l'auteur n'est responsable que s'il a commis une faute et est excusé dans les autres cas. Deuxièmement, dans le cas d'activités particulièrement dangereuses, comme la production d'énergie nucléaire, une responsabilité dite objective a été développée. Dans ce cas, la faute de l'opérateur n'est pas un facteur déterminant pour le déclenchement de la responsabilité. Au contraire, l'opérateur est responsable du seul fait qu'il s'engage dans une activité donnée pour tout dommage causé par cette activité même s'il n'a pas commis de faute ou n'a pas connaissance des défauts du produit en question. La responsabilité objective peut être distinguée de la responsabilité absolue par le fait qu'elle comprend un certain nombre de clauses d'exonération de l'opérateur. Celles-ci comprennent par exemple les situations de guerre, les catastrophes naturelles ou la faute d'un tiers. La responsabilité absolue est une responsabilité objective sans clause d'exonération.

Un régime de responsabilité permet d'indemniser différents types de dommages. Traditionnellement, la responsabilité s'est concentrée sur les dommages aux personnes et à la propriété. Plus récemment, le dommage environnemental a été considéré soit en tant que facteur causant un dommage à la propriété ou aux personnes soit en tant que dommage propre. Le dommage environnemental peut comprendre le coût des mesures de réparation de l'environnement, les pertes liées à la diminution possible de l'utilisation des ressources naturelles pour satisfaire aux besoins de base, les pertes liées au manque à gagner consécutif à la dégradation de l'environnement et le coût des mesures entreprises ou à entreprendre pour prévenir le dommage environnemental.

Le cas du dommage environnemental pose des problèmes particuliers en ce qui concerne la réparation du dommage. En effet, dans le cas d'un dommage qui

Par ex. Paul Martin v. Reynolds Metals Company, Supreme Court of Oregon, 29 juillet 1959, 342 P.2d 790 . 
n'est pas lié à un droit de propriété réel ou dans le cas d'un dommage qui est difficilement mesurable en terme de compensation financière, il est impératif de trouver d'autres moyens permettant de compenser le dommage. En pratique, la restauration de l'environnement semble être une des solutions les plus justes dans le cas où il n'y a pas de perte économique à proprement parler. Cette solution ne peut cependant pas être employée dans tous les cas puisque l'environnement affecté peut être trop endommagé pour pouvoir être restauré. C'est le cas par exemple des situations où l'activité dangereuse cause un dommage irréversible. Deux solutions se présentent alors: la création d'un environnement similaire dans un autre endroit ou une sanction pénale. Aucune de ces deux solutions ne sont optimales d'un point de vue environnemental et devraient constituer des exceptions pour certains cas exceptionnels.

\subsection{La responsabilité en droit international}

Le droit international a développé un certain nombre de règles concernant la responsabilité des Etats et la responsabilité civile qui constituent le point de départ pour un examen des questions de responsabilité dans le contexte du génie génétique. La responsabilité des Etats peut être engagée dans les cas où des activités ont des conséquences transfrontières néfastes pour d'autres Etats ou des zones de relevant pas d'une juridiction nationale ${ }^{4}$. Les problèmes potentiels comprennent, par exemple, la question de la responsabilité d'Etats qui envoient de l'aide alimentaire sous forme de semences transgéniques et la question de la responsabilité des Etats dans les cas où les plantes transgéniques d'un pays contaminent l'environnement d'un Etat voisin. Le droit international de la responsabilité étatique reste très général dans les principes qui sont reconnus. Le principe de base est que les Etats sont responsables des actes ou omissions qui sont contraires au droit international ${ }^{5}$. Des principes concernant les activités non contraires au droit international ont également été développés. En ce qui concerne le dommage couvert par les règles de responsabilité étatique, différents régimes prévoient tant des définitions restrictives ne couvrant que le dommage strictement environnemental que des définitions qui comprennent également le dommage aux ressources naturelles et le paysage. Il n'existe pas non plus de seuil spécifique de dommage qui déclenche la responsabilité mais en général le dommage doit être grave $^{6}$. Le droit international fait également une distinction entre les activités qui sont considérées ultra dangereuses et les autres activités. Les conséquences du déclenchement de la responsabilité étatique sont établies en général. Les Etats sont tenus de réparer le dommage causé par restitution, compensation et/ou satis-

Cf. Principe 2, Déclaration de Rio sur l'environnement et le développement, 14 juin 1992, Doc. ONU A/CONF.151/26/Rev.1 (Vol. 1), Annex I.

Commission du droit international, Projet d'articles sur la responsabilité de l'Etat pour fait internationalement illicite, Doc. ONU A/56/589 (2001).

6 Par ex. Article 2 de la Position commune (CE) No 58/2003 arrêtée par le Conseil le 18 septembre 2003 en vue de l'adoption de la directive 2003/.../CE du Parlement européen et du Conseil du... sur la responsabilité environnementale en ce qui concerne la prévention et la réparation des dommages environnementaux, Journal officiel de l'Union européenne 2003/C 277 E/02 (18 nov. 2003). 
faction. ${ }^{7}$ Ces principes généraux n'ont été que peu utilisés par les Etats qui ont en général préféré éviter l'utilisation des règles de la responsabilité étatique dans les cas de dommages environnementaux pour éviter la création de précédents pouvant se retourner contre eux plus tard. Une des rares exceptions concerne le cas d'un satellite soviétique qui s'écrasa dans le nord du Canada. La Convention concernant la responsabilité pour les objets spatiaux prévoit que les Etats ont une responsabilité absolue concernant les dommages causés par leurs objets spatiaux ${ }^{8}$. Dans ce cas, le Canada réclama des dommages à l'URSS pour les mesures de décontamination'. En somme, le droit international ne donne donc que des indications très générales concernant les conséquences possibles d'un dommage environnemental transfrontière causé par des organismes transgéniques. Les règles générales indiquent qu'un dommage significatif entraîne des conséquences juridiques mais les conséquences spécifiques ne peuvent être déterminées sur la base des règles existantes.

Les autres questions importantes dans le domaine de la responsabilité pour dommage à l'environnement causé par des organismes transgéniques concernent la responsabilité civile puisque la plupart des développements dans ce domaine sont produits par le secteur privé. Le droit international est plus développé dans ce domaine. Il existe plusieurs conventions qui comprennent des régimes sectoriels relativement développés de responsabilité civile pour des activités dangereuses, comme par exemple dans le domaine de l'énergie nucléaire, de la pollution par hydrocarbures et des déchets dangereux ${ }^{10}$. Ces différents traités proposent des régimes relativement similaires. Le régime de responsabilité civile prévu est en général fondé sur le principe qu'il s'agit d'une responsabilité dite objective ${ }^{1}$. Cela est lié à la reconnaissance qu'il s'agit d'activités dangereuses pour l'environnement qui méritent un traitement particulièrement strict. Les clauses d'exonération habituelles, telles que la guerre ou les catastrophes naturelles sont en général incluses ${ }^{12}$. Dans certains cas, une responsabilité pour faute pour toute personne qui cause ou contribue à causer un dommage par préméditation, imprudence ou négligence s'ajoute à la responsabilité objective ${ }^{13}$.

Les différents régimes divergent en ce qui concerne la possibilité pour la partie responsable de se retourner contre d'autres acteurs. Ainsi, le régime juridique pour l'énergie nucléaire ne permet aucun recours contre des tiers alors que le

7 Cour permanente de justice internationale, Affaire relative à l'usine de Chorzów, 13 septembre 1928, Recueil des arrêts, Série A, No 17.

8 Convention sur la responsabilité internationale pour les dommages causés par les objets spatiaux, Londres, 29 mars 1972, 961 Recueil des traités des Nations Unies 187.

9 Voir Canada: Claim against the USSR for Damage Caused by Soviet Cosmos 954, 18 International Legal Materials 899 (1979).

10 En ce qui concerne l'énergie nucléaire, voir Convention de Vienne relative à la responsabilité civile en matière de dommage nucléaire, Vienne, 21 mai 1963 telle qu'amendée par le Protocole du 12 septembre 1997, AIEA Doc. INFCIRC/566 [ci-après Convention de Vienne 1963/1997] et Convention sur la réparation complémentaire des dommages nucléaires, Vienne, 12 septembre 1997, IAEA Doc. INFCIRC/567.

11 Par ex. Protocole sur la responsabilité et l'indemnisation en cas de dommages résultant de mouvements transfrontières et de l'élimination de déchets dangereux, 10 décembre 1999, ONU doc. UNEP/CHW.5/29, Annexe III (1999) [ci-après Protocole de la Convention de Bâle].

12 Par ex. Article 4(5) du Protocole de la Convention de Bâle, n. 11 ci-dessus.

13 Par ex. Article 2(5) de la Convention de Vienne 1963/1997, n. 10 ci-dessus. 
Protocole à la Convention de Bâle prévoit un tel droit de recours ${ }^{14}$. Par ailleurs, la responsabilité est presque toujours limitée dans son montant et dans le temps ${ }^{15}$. Finalement, dans certains cas, comme dans le cas des traités sur l'énergie nucléaire, le régime de responsabilité civile prévoit une assurance obligatoire des opérateurs et une responsabilité subsidiaire de l'Etat. Au niveau des dommages couverts, les traités sur la responsabilité civile ont eu tendance à écarter du champ d'application des dommages strictement environnementaux tels que les dommages aux écosystèmes. Dans la plupart des cas, les dommages à l'environnement sont pris en compte au travers de la considération des dommages aux personnes, à la propriété et aux intérêts économiques. Cependant, les textes les plus récents prennent maintenant en compte les coûts engendrés par des mesures préventives et la restauration d'un environnement dégradé ${ }^{16}$.

Dans l'ensemble, les régimes de responsabilité constituent un essai de concilier la nature dangereuse des activités proposées et la volonté des Etats de promouvoir un certain type de développement technologique et industriel. Le régime adopté pour l'énergie nucléaire reconnaît par exemple la nécessité d'adopter des règles strictes pour la compensation d'un éventuel dommage causé par un incident radioactif et la nécessité de développer un système équitable et équilibré pour répartir les pertes entre les opérateurs. Le Protocole de Cartagena fait de même en adoptant des règles facilitant le commerce international des organismes transgéniques tout en prévoyant un cadre juridique strict basé sur le principe de précaution et en reconnaissant la nécessité de développer des règles sur la responsabilité.

Alors que les traités mentionnés ci-dessus concernent chacun un domaine particulier, il existe un traité régional négocié dans le cadre du Conseil de l'Europe (Convention de Lugano) qui concerne la responsabilité pour différents types de dommages à l'environnement ${ }^{17}$. Son objectif général est d'assurer une réparation adéquate des dommages résultant des activités dangereuses pour l'environnement. La définition des activités dangereuses comprend entre autres

\begin{abstract}
la production, la culture, la manipulation, le stockage, l'utilisation, la destruction, l'élimination, la libération ou toute autre opération concernant un ou plusieurs organismes génétiquement modifiés qui, en raison des propriétés de l'organisme, de sa modification génétique et des conditions dans lesquelles l'opération est réalisée, présentent un risque significatif pour l'homme, l'environnement ou les biens ${ }^{18}$.
\end{abstract}

La définition du dommage selon la Convention de Lugano est également intéressante dans la perspective de la biotechnologie. Le dommage comprend non seulement les pertes ou dommages résultant de l'altération de l'environnement mais également le décès ou des lésions corporelles ${ }^{19}$. Cependant, dans le contexte

\footnotetext{
Article 8 du Protocole de la Convention de Bâle, n. 11 ci-dessus.

Par ex. Articles 5 et 6 de la Convention de Vienne 1963/1997, n. 10 ci-dessus.

Par ex. Article 1(k) de la Convention de Vienne 1963/1997, n. 10 ci-dessus.

Convention sur la responsabilité civile des dommages résultant d'activités dangereuses pour l'environnement, Lugano, 21 juin 1993, Série des traités européens No 150 [Convention de Lugano].

18 Article 2(1) de la Convention de Lugano, n. 17 ci-dessus.

19 Les dommages à l'environnement sont pris en compte dans la limite des mesures de remise en état qui ont été effectivement prises ou qui le seront. Voir Article 2(7) de la Convention de Lugano, n. 17 ci-dessus.
} 
de la biotechnologie une réserve peut être faite par les Etats qui le désirent pour exclure le dommage causés par les organismes transgéniques quand l'état des connaissances scientifiques et techniques au moment de l'événement ne permettait pas de connaître l'existence des propriétés dangereuses de la substance ou le risque significatif que présentait l'opération concernant l'organisme ${ }^{20}$. Malgré les réserves possibles, la Convention de Lugano a une portée si significative que les Etats du Conseil de l'Europe se sont pour l'instant abstenus de la ratifier et seuls quelques uns l'ont signée ${ }^{21}$.

En somme, les régimes existants de responsabilité civile en droit international sont intéressants pour plusieurs raisons. Premièrement, le développement du droit international indique que les Etats ont préférés jusqu'à présent développer des règles de responsabilité spécifiques pour différents types d'activités plutôt que de développer des règles générales de responsabilité pour tous les dommages à l'environnement. Deuxièmement, la Convention de Lugano qui a tenté une approche plus générale ne semble pas remporter l'adhésion des Etats, ce qui semble indiquer une nécessité de continuer d'approcher les questions de responsabilité dans une perspective sectorielle pour l'instant. Troisièmement, il existe un cadre général pour la responsabilité étatique proposé par la Commission du droit international. Bien que les dispositions prévues ne soient pas spécifiques au dommage environnemental, elles peuvent fournir les principes de base dans le contexte de la biotechnologie. Quatrièmement, il apparaît que les questions de responsabilité civile et de responsabilité étatique doivent être traitées de façon séparée même si les principes généraux sont semblables dans les deux cas.

Le bref aperçu du droit international montre qu'un certain nombre de règles concernant la responsabilité pour dommage à l'environnement existent déjà en droit international. Cependant, ces règles tendent à être soit de nature générale, comme en ce qui concerne les règles de droit coutumier international, soit applicables seulement dans des contextes spécifiques tels que l'énergie nucléaire. Le seul traité qui traite de la responsabilité pour dommage à l'environnement en général et comprend la question des organismes transgéniques, la Convention de Lugano, est un traité régional qui n'est pas en vigueur.

\section{LA RESPONSABILITÉ DANS LE CONTEXTE DE LA BIOTECHNOLOGIE}

Les questions liées à la responsabilité dans le domaine de la biotechnologie méritent une analyse séparée. Certains aspects du sujet sont relativement proches de régimes juridiques existants, par exemple, en ce qui concerne certains aspects du dommage environnemental. La biotechnologie pose également un certain nombre de questions supplémentaires qui doivent être considérés pour obtenir une image complète des questions qui se posent. Les deux aspects considérés dans cette section sont la question des dommages socio-économiques, par exemple le dommage subi par les agriculteurs biologiques dont les champs sont contaminés

\footnotetext{
$20 \quad$ Article 35 de la Convention de Lugano, n. 17 ci-dessus.

21 Seuls neuf Etats ont signé la Convention jusqu'à présent.
} 
par des variétés transgéniques et les prétentions que les détenteurs de brevets sur des organismes transgéniques ont contre les agriculteurs.

\subsection{Dommage à l'environnement}

La première question qui se pose est la question du dommage causé par l'introduction d'organismes transgéniques dans l'environnement. Alors que peu de conséquences néfastes ont été recensées jusqu'à présent, les études récemment achevées au Royaume-Uni indiquent qu'il est au minimum impossible d'exclure l'éventualité de la survenance de dommages ${ }^{22}$. Les impacts possibles des organismes transgéniques comprennent: les dangers liés à l'instabilité du matériel génétique et la possibilité de changements ultérieurs des organismes transgéniques; le transfert de gènes à d'autres organismes; le potentiel pour des variétés transgéniques de surperformance qui contribuerait au déplacement et/ou à la disparition des espèces naturelles ${ }^{23}$.

Par certains aspects, le cas de la responsabilité pour dommage à l'environnement est le plus simple puisqu'il existe un certain nombre de règles existantes ou proposées qui peuvent être appliquées ou transposées au cas des organismes transgéniques. Cette section examine les questions liées à la responsabilité pour dommage à l'environnement causé par des organismes transgéniques en général et examine en particulier la loi suisse sur le génie génétique qui est à ce jour une des rares lois à prévoir un régime spécial de responsabilité pour la biotechnologie. Cette dernière ne constitue pas un modèle pour le développement du droit international ou les autres pays mais elle est néanmoins significative étant donné qu'il s'agit d'un texte de loi adopté par un pays qui a des intérêts commerciaux importants dans les domaine de la biotechnologie.

Passant maintenant aux conditions générales de la responsabilité qui s'appliquent aussi dans le cas des organismes transgénique, une première condition est que la responsabilité ne peut être engagée que si les acteurs sont directement identifiables. Cela signifie qu'un dommage dont la source ne peut pas être identifiée ne peut être compensé selon les règles de la responsabilité civile ${ }^{24}$. Dans ce cas, il est nécessaire de recourir à d'autres mécanismes de compensation, comme l'assurance. Les organismes transgéniques peuvent en principe donner lieu à des questions importantes concernant l'identification de l'inventeur et du producteur dans les cas de contamination environnementale où il peut être difficile d'identifier le lien entre l'auteur et le dommage. En pratique, grâce au fait que le développement du génie génétique est conditionné en partie par l'obtention de brevets et autres droits de propriété intellectuelle, il devrait être possible dans la plupart des cas d'identifier l'auteur du dommage.

La question suivante concerne la définition du dommage environnemental. Pour être compensable, le dommage ne doit pas être diffus et une relation entre les

22 Par ex. Maria Burke, GM Crops - Effects on Farmland Wildlife (Londres: Farmscale Evaluations Research Team and the Scientific Steering Committee, 2003).

23 Par ex. Commission de la science, de l'éducation et de la culture - Conseil des Etats, 00.008 e Projet Gen-Lex (droit de la responsabilité civile), Rapport du 27 août 2001.

24 Voir en général sur cette question, Alexandre Kiss \& Jean-Pierre Beurier, Droit international de

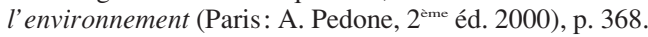


conséquences négatives pour l'environnement et l'introduction des organismes transgéniques doit être établie. Hormis ces conditions de base, l'étendue du dommage compensable peut être plus ou moins vaste suivant que le coût de mesures préventives pour empêcher les atteintes à l'environnement et le coût de mesures de restauration de l'environnement sont pris en compte ou non. Certains traités prévoient que tout dommage déclenche la responsabilité des auteurs du dommage $^{25}$. Dans le cas des organismes transgéniques, une solution aussi étendue risque de rendre le génie génétique très peu attractif. Une solution plus limitée qui prévoit que le dommage causé doit être dû aux propriétés des organismes transgéniques, leur reproduction ou la modification des organismes, ou le transfert du matériel génétique modifié de ces organismes est probablement plus adaptée pour les Etats qui veulent maintenir un équilibre entre les mesures d'incitation et de protection. C'est une solution choisie par exemple par la loi suisse qui prévoit qu'une atteinte est 'tout effet nuisible ou incommodant exercé par les organismes génétiquement modifiés sur l'être humain, sur les animaux ou sur l'environnement $^{26}$.

Deux autres problèmes importants doivent être mentionnés. Le genre de risque causé par l'activité ayant des conséquences potentiellement néfastes pour l'environnement influence en général le degré de vigilance exact requis des promoteurs. Deux types principaux de responsabilité peuvent être envisagés. Dans le premier cas où le législateur estime que le risque propre à l'activité est relativement faible, seule une faute causée soit intentionnellement soit par négligence entraîne la responsabilité de l'auteur. Dans le cas où l'activité envisagée est considérée dangereuse pour l'environnement, le législateur peut alors décider que la responsabilité de l'auteur est encourue qu'il y ait faute ou non. Dans ce cas de responsabilité dite objective, l'auteur ne peut se décharger que dans des situations 'extraordinaires' telles que la guerre ou des actes commis par une tierce personne.

Au niveau international, la question de la nature des risques inhérents à l'introduction d'organismes transgéniques dans l'environnement n'a pas été résolue et les négociateurs d'un régime de responsabilité dans le cadre du Protocole sur la biosécurité devront y répondre dans les quatre ans à venir. Au niveau national, la Suisse qui est un des rares pays à avoir adopté un régime de responsabilité pour la biotechnologie a clairement choisi une forme de responsabilité objective ${ }^{27}$. Ainsi, la loi suisse prévoit que, sans exception

[t]oute personne soumise au régime de la notification ou de l'autorisation qui utilise des organismes génétiquement modifiés en milieu confiné, qui dissémine de tels organismes dans l'environnement à titre expérimental ou qui les met sans autorisation en circulation, répond des dommages causés par cette utilisation et dus à la modification du matériel génétique de ces organismes ${ }^{28}$.

5 Par ex. Article 3 de la Convention internationale sur la responsabilité civile pour des dommages dus à la pollution par les hydrocarbures, Londres, 27 novembre 1992.

26 Article 5(3) de la Loi fédérale suisse sur l'application du génie génétique au domaine non humain, 21 mars 2003, Recueil systématique 814.91 [ci-après Loi sur le génie génétique].

27 Selon la loi, seules la force majeure ou à une faute grave du lésé ou d'un tiers permettent au titulaire d'une autorisation de se décharger de sa responsabilité; Article 30(8) de la Loi sur le génie génétique, n. 26 ci-dessus.

28 Article 30(1) de la Loi sur le génie génétique, n. 26 ci-dessus. 
La loi suisse prévoit également que le titulaire de l'autorisation d'utilisation ou de dissémination d'organismes transgéniques est seul responsable du dommage et ne peut se retourner contre des tiers que dans les cas où des utilisateurs ont utilisés les organismes transgéniques de façon inadéquate. Dans le cas où le dommage est causé par une interaction entre plusieurs organismes transgéniques, le titulaire de l'autorisation ne répond du dommage que si l'organisme en question est défectueux ${ }^{29}$. Cependant, le titulaire reste responsable de défauts qui n'auraient pas été détectés au moment de la mise en circulation parce que les connaissances scientifiques n'étaient pas suffisantes. Le principe de précaution trouve donc ici une application indirecte importante dans une version forte qui met à la charge du détenteur de l'autorisation le fardeau du dommage dans le cas où il survient à cause d'une incapacité de l'autorité à évaluer les risques au moment de délivrer l'autorisation.

Un autre aspect important de la responsabilité dans le domaine de la biotechnologie - tout comme dans d'autres domaines comme l'énergie nucléaire concerne la période durant laquelle la responsabilité peut être engagée. Etant donné la nature aléatoire des conséquences négatives de l'introduction de plantes transgéniques dans l'environnement, la loi suisse a par exemple accepté une période de 30 ans à partir du moment où l'événement dommageable a eu lieu ${ }^{30}$.

Les conséquences de la responsabilité dans le cas de dommage à l'environnement causés par des organismes transgéniques comprennent un devoir de réparation. Selon la loi suisse, la réparation des dommages à l'environnement comprend les mesures prises pour remettre en état les éléments de l'environnement détruits ou détériorés ou le coût des mesures prises pour les remplacer par un équivalent ${ }^{31}$. Un des problèmes importants qui devra être pris en compte au niveau international encore plus qu'au niveau national est la question du dommage causé à des zones qui ne sont pas l'objet de droits réels au niveau national ou des zones qui ne sont pas sous contrôle souverain d'un Etat au niveau international. En effet, le droit de la responsabilité n'est encore que peu développé en ce qui concerne la réparation des atteintes à l'environnement en tant que tel. Cela est dû au fait qu'il manque dans la plupart des cas un ombudsman qui soit chargé d'actionner les parties fautives pour réparation de l'environnement quand aucune personne ou Etat n'est directement affecté. La loi suisse prévoit dans ce genre de situations que les collectivités publiques sont compétentes pour prendre les mesures nécessaires contre l'auteur du dommage. Au niveau international, il conviendra d'appliquer un des principes de base du droit international de l'environnement qui prévoit que les Etats doivent s'efforcer de faire en sorte que les activités entreprises sur leur

29 La loi détermine qu'un 'organisme génétiquement modifié est considéré comme défectueux lorsqu'il n'offre pas la sécurité que l'on est en droit d'attendre compte tenu des circonstances; il y a lieu notamment de prendre en compte: a. la manière dont il est présenté au public; b. l'utilisation qu'on est raisonnablement en droit d'attendre; c. la date de sa mise en circulation'; Article 30(5) de la Loi sur le génie génétique, n. 26 ci-dessus.

3o Une période de trois ans à partir du moment où le lésé a eu connaissance du dommage et de l'identité de la personne responsable est également prévue à l'Article 32 de la Loi sur le génie génétique, n. 26 ci-dessus.

31 Article 31 de la Loi sur le génie génétique, n. 26 ci-dessus. 
territoire ne causent pas de dommage aux autres Etats ou aux zones ne relevant d'aucune juridiction territoriale.

Une alternative à la réparation est l'imposition de sanctions pénales pour punir les auteurs du dommage ${ }^{32}$. Le droit brésilien reflète par exemple une approche qui privilégie une approche pénale ${ }^{33}$. Bien que l'emprisonnement ou des amendes puissent constituer un moyen de dissuasion importants, il semble important dans le cas du dommage environnemental des organismes transgéniques de prévoir un régime juridique qui prévoit en premier lieu la réparation du dommage environnemental par l'auteur du dommage plutôt que de remettre ce fardeau indirectement à la communauté publique.

Finalement, il convient de noter qu'il existe un certain nombre de problèmes qui n'ont pas encore été envisagés dans les règles existantes ou prévues. Ainsi, l'un des cas problématiques que la communauté internationale devra aborder est celui de la relation entre le développement du génie génétique indirectement encouragé par le Protocole sur la biosécurité et le principe général de conservation prévu par la Convention sur la biodiversité qui implique, par exemple, la nécessité de prendre des mesures de protection spécifiques pour les centres de diversité biologique. La question se pose donc de savoir si des règles particulières devraient être rédigées pour préserver de la contamination possible par organismes transgéniques les zones qui sont des centres de diversité pour les cultures de base telles que le ris, le maïs et le blé. Il semble approprié de prévoir tant au niveau international qu'au niveau national des règles très strictes de responsabilité pour empêcher la contamination de toute zone qui pourra offrir dans le futur une source potentielle de diversité biologique contre de nouveaux problèmes qui affecteront les cultures vivrières.

\subsection{Dommage socio-économique}

La question de la responsabilité dans le domaine du génie génétique va plus loin que les problèmes mentionnés dans le contexte du dommage environnemental. En effet, l'introduction d'organismes génétiquement modifiés dans l'environnement a la capacité de causer non seulement des conséquences négatives pour l'environnement mais peut également avoir un certain nombre de conséquences socio-économiques négatives qui doivent également être prises en compte.

Les dommages socio-économiques peuvent être liés à un dommage environnemental et en pratique seront souvent liés directement ou indirectement à des conséquences négatives pour l'environnement. Cependant, d'un point de vue des principes, la question de la responsabilité pour dommage socio-économique est séparée et il existe des situations où un dommage socio-économique ne sera pas associé à un dommage environnemental. Plusieurs exemples peuvent être donnés pour illustrer le dommage socio-économique dans le contexte de la biotechnologie.

Par ex. Convention sur la protection de l'environnement par le droit pénal, Strasbourg, 4 novembre 1998, Série des traités européens No 172.

33 Article 13, Brésil: lei de biossegurança, 1995. 
L'un des exemples les plus clairs concerne les cas où les variétés transgéniques contaminent des champs où poussent des récoltes biologiques. Dans ce cas, même si le dommage environnemental n'est pas confirmé, il y a un dommage économique immédiat pour les agriculteurs dont les cultures biologiques vendues à un prix majoré ne peuvent plus prétendre à la dénomination biologique qui n'autorise pas la présence d'organismes transgéniques ${ }^{34}$. Le récent rapport de la Commission biotechnologique britannique a chiffré les pertes potentielles d'agriculteurs biologiques qui perdraient, par exemple, environ 500 livres sterling par hectares ${ }^{35}$. Au Canada, les cultivateurs de colza biologique du Saskatchewan tentent actuellement de poursuivre en justice deux multinationales qui produisent du colza transgénique. Les agriculteurs accusent Monsanto et Aventis d'avoir causé la contamination de leurs champs qui ne leur permet plus de produire du colza qui peut être certifié comme étant biologique ${ }^{36}$.

Les autres cas de figure concernent principalement les pays du Sud. Premièrement, dans le cas où l'introduction de variétés transgénique contribue au déplacement ou à la disparition d'autres variétés, il y a un dommage environnemental. Ce dommage peut être associé à un dommage socio-économique dans tous les cas où la variété locale qui disparaît est une variété utilisée dans l'agriculture de subsistance et donc directement liée à la satisfaction des besoins de base en alimentation.

Deuxièmement, il peut y avoir des situations où un dommage socio-économique n'implique pas de dommage environnemental comme dans le cas de la substitution de variétés non transgéniques par des variétés transgéniques dans les pays du Nord. Dans les cas où les variétés non transgéniques sont importées, par hypothèse de pays du Sud, une substitution par variété transgénique aurait un impact direct sur les revenus d'exploitation des pays producteurs concernés ${ }^{37}$.

Troisièmement, d'un point de vue général, l'introduction de variétés transgéniques devrait être jugée en fonction de la situation actuelle. Si les nouvelles variétés contribuent à réduire l'insécurité alimentaire, elles ont un impact positif. Si elles augmentent l'insécurité alimentaire, elles causent un net dommage socioéconomique. Etant donné que les variétés transgéniques sont par hypothèse plus chères que les variétés locales puisqu'elles sont protégées par des brevets ou autres droits de propriété intellectuelle, l'augmentation du prix doit être accompagnée par des bénéfices socio-économiques pour le pays et les individus les plus pauvres de la société qui n'ont pas accès à des rations alimentaires suffisantes. Dans la situation d'un pays du groupe des pays les moins avancés, l'introduction de variétés transgéniques plus chères que les variétés locales ne peut être justifiée

Par ex. Department for Environment, Food and Rural Affairs, Compendium of UK Organic Standards (Version 3.5, mars 2003).

35 Agriculture and Environment Biotechnology Commission, GM Crops? - Coexistence and Liability (London: Biotechnology Commission, novembre 2003).

${ }^{36}$ L. Hoffman \& D. Beaudoin et Monsanto \& Aventis, Statement of claim, Saskatoon, 10 janvier 2002.

37 Propositions du groupe africain sur les considérations socio-économiques, in Groupe de travail spécial à composition non limitée sur la prévention des risques biotechnologiques, Compilation des vues des Gouvernements sur la teneur du futur Protocole, ONU Doc. UNEP/CBD/BSWG/2/2 (1997). 
du point de vue socio-économique que si le produit final a des caractéristiques nutritionnelles améliorées sans causer de dommage à l'environnement ou à la santé. Dans le cas contraire, la société en général n'y trouve pas un bénéfice suffisant sur le plan socio-économique. Le problème identifié ici peut survenir tant dans le cas où la production de variétés transgéniques est organisée au niveau national que dans le cas où des produits transgéniques sont importés, par exemple sous forme d'aide alimentaire.

\subsection{Dommage subi par le détenteur de droits de propriété intellectuelle}

Dans la plupart des domaines où se pose la question de la responsabilité pour dommage à l'environnement, les problèmes qui se posent concernent la responsabilité du promoteur de l'activité en question pour les dommages causés. Dans le cas du génie génétique cependant l'histoire récente indique qu'un nouveau type de dommage ou plus exactement de 'contre dommage' a fait son entrée. Ce nouveau type de problème est lié aux incitations au développement du génie génétique qui comprennent entre autres la possibilité d'obtenir des droits de propriété intellectuelle - en particulier des brevets - sur les organismes transgéniques pour les compagnies engagées dans la biotechnologie.

Le cas d'école est celui d'une entreprise détentrice de brevets sur des organismes transgéniques qui poursuit en justice un agriculteur pour violation de ses droits. En tant que tel, une action en justice pour violation d'un brevet est une affaire courante qui est une conséquence normale du droit de monopole offert au détenteur. Dans le cas d'organismes transgéniques, cependant, d'autres facteurs doivent être pris en compte pour décider des prétentions des diverses parties.

Pour l'instant, les cas d'applications semblent limités à l'Amérique du Nord mais les questions qui se posent sont d'une importance cruciale pour le développement de régimes juridiques tant dans le domaine de la propriété intellectuelle que de la biosécurité. Parmi les décisions prises par les tribunaux, l'affaire Schmeiser est la plus significative à ce jour. Dans cette affaire, Monsanto a intenté une action contre Percy Schmeiser pour violation d'un de ses brevets sur le colza transgénique. La plainte reprochait à $\mathrm{M}$. Schmeiser d'avoir contrevenu au brevet en exploitant, reproduisant et créant des gènes, des cellules, ainsi que des graines et des plants de canola contenant des gènes et des cellules revendiqués dans le brevet et en vendant les graines de canola récoltées sans avoir obtenu l'autorisation ou une licence de Monsanto. Le juge de première instance délivra en 2001 une décision en faveur de Monsanto ${ }^{38}$, confirmée en appel en $2002^{39}$. Deux aspects principaux de la décision retiennent l'attention. D'une part, le juge a cherché à définir les conditions sous lesquelles un agriculteur est responsable de l'apparition de semences brevetées sur son terrain. Il a indiqué que

le producteur dont le champ contient des graines ou des plants qui sont issus de semences qui y ont été déversées ou qui proviennent de plants fauchés sur la terre d'un voisin ou même qui sont le fruit de la germination de pollens transportés dans son champ par des insectes, des

Monsanto Canada et Percy Schmeiser, 29 mars 2001, [2001] 3 Recueil des arrêts de la Cour fédérale du Canada D-36.

39 Monsanto Canada et Percy Schmeiser, 4 septembre 2002, [2003] 2 Recueil des arrêts de la Cour fédérale du Canada 165 [ci-après Monsanto et Schmeiser (appel)]. 
oiseaux ou par le vent, peut être propriétaire des graines ou des plants qui se trouvent sur sa terre même si ce n'est pas lui qui a les a plantés. Il n'a toutefois pas le droit d'exploiter le gène breveté ou la graine ou la plante contenant la cellule ou le gène brevetés ${ }^{40}$.

Le juge McKay indiqua clairement que les droits du détenteur du brevet prévalent sur les droits réels du propriétaire terrien et que ce dernier peut être contraint de payer une redevance au détenteur du brevet même sans faute de sa part ${ }^{41}$.

Le juge va plus loin et détermine que le défendeur doit payer une redevance à Monsanto même s'il n'a pas profité des qualités spécifiques du produit breveté. Il conclut que

M. Schmeiser savait ou peut être présumé avoir su [que les variétés cultivées étaient] résistantes au Roundup. Ces graines ont poussé et les plants ont finalement été récoltés puis vendus. A mon avis, le fait que cette récolte ait ou non été traitée au Roundup au cours de sa période de croissance est sans importance. La croissance de la semence qui reproduit le gène et la cellule brevetés et la vente de la récolte constituent une appropriation de l'essence même de l'invention et une utilisation sans permission de cette semence. En agissant ainsi, les défendeurs ont violé les droits que les demanderesses possédaient en vertu de leur brevet ${ }^{42}$.

En suivant la logique du juge McKay, M. Schmeiser doit une redevance à Monsanto même si dans le cas particulier il n'a pas profité des qualités spécifiques des semences transgéniques qui sont prévues pour résister à l'application de l'herbicide Roundup fabriqué par Monsanto.

Le jugement d'appel va plus loin dans la réflexion concernant la présence potentiellement fortuite de semences brevetées. Les juges acceptent que dans un cas où le propriétaire ignore tout de la qualité particulière d'une semence transgénique, '[i]l y a beaucoup de force à l'argument selon lequel il serait inéquitable d'accorder à Monsanto une réparation par suite de la contrefaçon lorsqu'il y a des repousses spontanées ${ }^{\prime 3}$. Ils refusent cependant de se prononcer sur ce point. Leur décision est finalement basée sur le fait qu'en suivant le juge de premier instance les juges estiment que M. Schmeiser savait ou aurait dû savoir que les plants litigieux résistaient au glyphosate lorsqu'il avait conservé leurs graines en 1997 et qu'il les avait semées l'année suivante ${ }^{44}$.

Le jugement Schmeiser pose un certain nombre de questions importantes pour le développement de régimes de responsabilité dans le contexte du génie génétique. Premièrement, la question se pose de savoir comment les droits de propriété intellectuelle doivent être considérés face aux droits réels ou même aux droits fondamentaux tels que le droit à l'alimentation. Le jugement se permet un important raccourci en évitant une décision sur la relation entre droits réels et droits de

40 Monsanto et Schmeiser, n. 38 ci-dessus, $\$ 92$.

${ }_{41}$ Monsanto et Schmeiser (appel), n. 39 ci-dessus au $\$ 51$ confirme ce point en indiquant qu'il 'n'existe aucune décision faisant autorité à l'appui de la thèse selon laquelle le droit de propriété existant sur un plant doit nécessairement l'emporter sur les droits que possède le breveté sur un gène contenu dans le plant. Au contraire, la jurisprudence présente un certain nombre d'exemples dans lesquels les droits de propriété existant sur un bien sont compromis dans la mesure nécessaire pour protéger le monopole conféré par la loi au breveté'.

42 Monsanto et Schmeiser, n. 38 ci-dessus, $\$ 123$.

43 Monsanto et Schmeiser (appel), n. 39 ci-dessus, $\$ 56$.

44 Monsanto et Schmeiser (appel), n. 39 ci-dessus, $\$ 58$. 
propriété intellectuelle et en se concentrant sur la question de la contrefaçon du brevet. Dans le contexte du droit des brevets, une telle conclusion est en général acceptable car il est couramment admis qu'un défendeur peut être responsable de la violation d'un brevet même sans avertissement et que la contrefaçon involontaire ou par inadvertance ne constitue pas une excuse valable ${ }^{45}$. Dans le cas des semences transgéniques, cependant, d'autres aspects doivent être pris en compte. D'une part, au niveau du droit des brevets, si la possession n'est pas intentionnelle et que les caractéristiques propres de l'invention ne sont pas exploitées, il semble que les principes habituels ne devraient pas s'appliquer. Par ailleurs la question doit être examinée en prenant en compte les autres facteurs qui entrent en jeu. Dans ce cas, on assiste à un conflit entre les principes du droit de la propriété intellectuelle et les principes de base des droits réels qui protègent le propriétaire terrien. Le droit coutumier prévoit entre autres des principes qui interdisent l'entrée non autorisée sur la propriété et le dommage à la propriété. Aux Etats-Unis, la jurisprudence déjà ancienne reconnaît que même une intrusion causée par des particules microscopiques peut constituer un délit ${ }^{46}$. Ainsi, même si le juge doit arriver à la conclusion que les principes coutumiers ne s'appliquent pas, cette discussion n'a pas été entreprise dans le cas Schmeiser et la décision est donc déficiente au niveau du raisonnement juridique. De surcroît, si la jurisprudence Schmeiser devait être acceptée par la Cour suprême du Canada, elle pourrait avoir des conséquences incalculables non seulement pour les agriculteurs mais pour toutes les entités juridiques contrôlant des terres. En suivant le juge McKay, M. Schmeiser doit non seulement être tenu responsable de la perte économique subit par Monsanto mais il serait également tenu responsable pour le dommage environnemental qui pourrait être créé par les organismes transgéniques. Dans une perspective plus large, cela signifie également que toute entité juridique qui a le contrôle de terres se retrouve responsable pour toutes les conséquences dues à l'intrusion d'organismes transgéniques. Cela pourrait par exemple signifier qu'un gouvernement tel que le gouvernement du Canada se verrait responsable des dommages causés à l'environnement par des organismes transgéniques brevetés à une entreprise privée pour tout dommage causé aux espaces publics et espaces non soumis à des droits réels privés. Il convient aussi de noter que si ces principes sont adoptés, tout propriétaire deviendrait responsable pour tout dommage causé à l'environnement par toute substance déversée sur leurs terres par des tiers.

Un autre problème important soulevé par le cas Schmeiser concerne la question de l'épuisement des droits de propriété intellectuelle. Au niveau international, il n'existe pas de consensus entre Etats. Certains estiment que le détenteur du brevet épuise ses droits après la première mise en circulation alors que d'autres estiment que la première mise en circulation dans un pays n'épuise pas les droits du détenteur dans les autres pays. La question de l'épuisement des droits n'est de loin pas théorique et peut avoir des impacts très importants sur la réalisation des

Par ex. Roger D. Blair \& Thomas F. Cotter, 'Strict Liability and its Alternatives in Patent Law', 17 Berkeley Technology Law Journal 799 (2002).

46 Martin v. Reynolds Metal Company, Supreme Court of Oregon, 29 juillet 1959, 342 Pacific Reporter-Second Series 790. 
besoins de base, par exemple dans le cas des produits pharmaceutiques ${ }^{47}$. En l'espèce, la jurisprudence nord-américaine accepte les clauses restrictives imposées par les détenteurs de brevets aux acheteurs qui leur garantissent un droit qui s'étend à la récolte produite grâce aux semences transgéniques. Ainsi, les restrictions imposées par Monsanto aux Etats-Unis à l'utilisation par les agriculteurs du produit de leur récolte sont jugées comme des clauses contractuelles qui ne laissent pas de place à l'application de la doctrine de l'épuisement des brevets ${ }^{48}$. Plus généralement, la récente décision McFarling indique spécifiquement que les clauses contractuelles imposées aux agriculteurs ne sont pas illégales puisqu'il n'y a aucune raison qu'un acheteur désirant acheter un produit d'une qualité supérieure doive s'attendre à ce que le producteur lui offre des conditions qui lui sont favorables.

\section{VERS LE DÉVELOPPEMENT DE RÈGLES SUR LA RESPONSABILITÉ POUR LA BIOTECHNOLOGIE}

Le développement de règles de responsabilité dans le domaine du génie génétique pose un certain nombre de problèmes juridiques, socio-économiques et politiques. Au niveau international, les Etats parties aux Protocole sur la biosécurité ont un mandat clair qui leur enjoint de commencer les négociations pour des règles sur la responsabilité dès 2004. Cela n'empêche pas une résistance importante. L'un des arguments mis en avant par les opposants est qu'il est inutile de tenter de développer un régime spécial de responsabilité pour la biosécurité et qu'il serait préférable de développer tant au niveau national qu'international des règles générales pour la responsabilité pour tout dommage à l'environnement ou à la biodiversité dans le contexte de la Convention sur la biodiversité ${ }^{49}$.

Il existe cependant un bon nombre de raisons pour développer des règles de responsabilité spécifiques à la biotechnologie tant au niveau international qu'au niveau national. En général, les organismes transgéniques n'ont pas été certifiés jusqu'à présent n'avoir aucun impact négatif environnemental et socio-économique. En application du principe de précaution, il est donc nécessaire de développer un régime juridique de responsabilité capable de faire face à toutes les éventualités même si la technologie prouve finalement n'avoir aucun effet négatif. De plus, un régime spécifique pour le génie génétique est nécessaire parce qu'un régime général de dommage à l'environnement ne suffirait pas à couvrir

${ }^{47}$ Pour plus de détails sur la question de l'épuisement et des importations parallèles, voir Frederick M. Abbott, 'First Report (Final) to the Committee on International Trade Law of the International Law Association on the Subject of Parallel Importation', 1 Journal of International Economic Law 607 (1998).

48 Par ex. Monsanto et William Trantham, United States District Court - W.D. Tennessee - Western Division, 16 août 2001, 156 West's Federal Supplement - Second Series 855 and Monsanto v McFarling, United States Court of Appeals, Federal Circuit, 23 août 2002, 302 West's Federal Reporter - Third Series 1291.

49 Par ex. Malcolm Forster \& Daniel Lawrence, 'The Cartagena Protocol: Moves Towards Establishing an International Liability Regime for Living Modified Organisms', 15 Environmental Law \& Management 5 (2003). 
toutes les situations envisagées dans la section précédente. Au niveau international, le droit international de l'environnement, dû entre autres au manque de centralisation qui le caractérise, a généralement évolué de façon sectorielle comme le montrent le développement de régimes de responsabilité spécifiques pour les hydrocarbures, les déchets dangereux ou l'énergie nucléaire. La spécificité des problèmes rencontrés dans le cas de la biotechnologie identifiés ci-dessus démontrent qu'un régime spécifique pour la biotechnologie est nécessaire au vu des problèmes spécifiques qui surviennent, ce que le processus législatif en Suisse confirme $^{50}$. De plus, si l'Article 27 du Protocole mentionne la question de la responsabilité uniquement dans le contexte transfrontière, c'est entre autres parce que l'intervention du droit international est nécessaire au niveau des rapports entre Etats ${ }^{51}$. Il n'est pas inusuel que le droit international laisse aux Etats le soin de régler leurs problèmes internes séparément. Dans le cas du génie génétique, il est nécessaire que les Etats développent en parallèle des règles au niveau international et au niveau national. Les premières doivent régler toutes les questions qui ressortent directement des mouvements transfrontières réglementés par le Protocole sur la biosécurité. Les deuxièmes doivent régler toutes les autres situations.

Les sections précédentes ont indiqué qu'hormis les règles définies par la Commission du droit international qui ne sont ni obligatoires ni spécifiques aux questions environnementales, il n'existe au mieux que le Convention de Lugano qui aborde le dommage environnemental dans son ensemble. Les seuls régimes de responsabilité adoptés au niveau international sont tous sectoriels et doivent donc être adaptés aux problèmes spécifiques posés par le génie génétique. Ce point est l'objet de débats au niveau international puisqu'il est parfois contesté que des régimes de responsabilité développés pour des activités ultra dangereuses puissent offrir un modèle approprié pour le génie génétique ${ }^{52}$. En fait, il y a d'une part un nombre croissant d'études indiquant le potentiel de contamination de l'environnement par des organismes transgéniques et par ailleurs on constate qu'un pays comme la Suisse a clairement fait le choix de suivre les modèles proposés pour les activités ultra dangereuses en application du principe de précaution.

Un régime de responsabilité doit avoir des buts bien définis pour prendre en compte tous les aspects pertinents de la question. Ceux-ci peuvent comprendre la protection de l'environnement, des animaux et de la santé humaine ainsi que la conservation de la biodiversité de la fertilité des sols et le maintien de l'intégrité des organismes vivants. Au delà des questions purement environnementales et sanitaires, un certain nombre d'objectifs socio-économiques sont également à prendre en compte. Ils comprennent la garantie du droit à l'alimentation et l'amélioration de

so Il est notable qu'en Suisse, un premier projet prévoyait d'introduire de nouvelles dispositions sur la biosécurité dans la Loi sur la protection de l'environnement. Finalement, le Parlement décida qu'il était nécessaire d'adopter une loi distincte entre autres parce qu'il était nécessaire d'adopter un régime de responsabilité spécifique au génie génétique pour tenir compte des intérêts des différents secteurs intéressés, soit les secteurs agricole et sylvicole d'une part et la recherche et l'industrie d'autre part. Voir Commission de la science, de l'éducation et de la culture - Conseil des Etats, 00.008 e Modification de la loi sur la loi protection de l'environnement (LPE) [loi sur le génie génétique, LGG], Rapport du 30 avril 2001.

${ }^{51} \quad C f$. Foster \& Lawrence, n. 49 ci-dessus, p. 6.

$52 \quad$ Foster \& Lawrence, n. 49 ci-dessus, p. 5. 
l'accès à la nourriture pour toutes les situations où les besoins de base ne sont pas satisfait. Dans les autres cas, un des buts est de permettre aux consommateurs le libre choix entre les produits transgéniques et non transgéniques. Enfin, le régime de responsabilité dans le domaine du génie génétique doit prendre position en ce qui concerne les limites du droit des brevets et déterminer dans le cadre des buts généraux énoncés ci-dessus les responsabilités des différents acteurs parties.

Différentes instruments juridiques peuvent être utilisés pour atteindre les buts visés. Le droit anglo-saxon offre en particulier des instruments de droit coutumier qui permettent de régler les conséquences d'actes délictuels sans avoir besoin de passer par des règles de responsabilité spécifiques. Comme la discussion du cas Schmeiser le montre, les problèmes liés à l'entrée non autorisée sur la propriété et le dommage à la propriété peuvent être réglés de cette façon. Cependant, le droit coutumier a une limite importante puisqu'il permet au mieux de régler les problèmes surgissant entre voisins. Ainsi, s'il est possible de prétendre selon ces règles à des dommage d'un agriculteur voisin pour contamination génétique, il serait très difficile d'appliquer les mêmes règles au détenteur du brevet concernant la dite modification génétique. Il est également admis qu'une personne entreprenant une activité particulièrement dangereuse a une responsabilité objective. L'interprétation habituelle des facteurs permettant de juger si une activité est particulièrement dangereuse pourrait mener à conclure qu'une entreprise qui produit volontairement un organisme ayant la capacité de se reproduire facilement doit supporter le coût des mesures nécessaires pour contrôler sa dissémination. En somme, il existe des principes intéressants pour régler certains problèmes concernant les dommages causés par les organismes transgéniques mais en l'état actuel du droit, le droit coutumier ne peut se substituer complètement à un régime de responsabilité civile qui devra aussi prendre en compte des aspects plus larges tels que les problèmes de santé publique, de protection du droit à l'alimentation, et de la protection de l'environnement dans les espaces communs. Un régime de responsabilité civile modelé d'après les règles existantes pour les activités dangereuses semble donc être l'instrument juridique le plus apte à réaliser les buts recherchés.

Jusqu'à présent, on constate que peu de pays du Sud ont cherché à développer des règles spécifiques de responsabilité pour le génie génétique, y compris des pays comme l'Inde où les premiers efforts pour réglementer les activités dans le domaine de la biotechnologie du point de vue de la biosécurité datent déjà de $1989^{53}$. L'Inde est un exemple intéressant puisque les questions de responsabilité pour dommage à l'environnement ont probablement fait l'objet d'une attention plus importante que dans tout autre pays du Sud suit à la tragédie de l'accident d'une usine de Union Carbide à Bhopal. Bien que dans le cas particulier de l'accident de Bhopal, Union Carbide n'ait jamais accepté sa responsabilité, la tragédie de 1984 mena le législateur indien et encore plus les tribunaux à s'intéresser aux questions afférentes au dommage environnemental ${ }^{54}$. Les développements des

s3 Ministry of Environment and Forests, Rules for the Manufacture, Use, Import, Export and Storage of Hazardous Micro Organisms Genetically Engineered Organisms or Cells, 5 décembre 1989.

${ }^{54}$ Par ex. Public Liability Insurance Act, 1991 et M.C. Mehta et Union of India (Shriram Gas Leak case), (1987) 1 Supreme Court Cases 395. La jurisprudence Shriram Gas Leak n'a pas été suivie dans le cas concernant la tragédie de Bhopal. Voir Union Carbide Corporation et Union of India (1991) 4 Supreme Court Cases 584. 
dernières deux décennies indiquent que le droit indien est aujourd'hui relativement développé en ce qui concerne la compensation du dommage environnemental $^{55}$. Par contre, le régime de responsabilité général pour dommage environnemental reste rudimentaire. L'exemple indien indique qu'il n'est pas possible d'escompter que les règles en vigueur actuellement soient suffisantes pour aborder les problèmes de responsabilité liés aux organismes transgéniques. Etant donné que l'Inde figure parmi les pays les plus avancés dans le développement de règles dans ce domaine, il apparaît important que chaque pays en développement prépare un cadre juridique pour faire face à toutes la éventualités liées à l'introduction d'organismes transgéniques sur son territoire.

Dans la plupart des pays, les questions de responsabilité seront principalement liées à des mouvements transfrontières puisque seul un petit nombre de pays a la capacité institutionnelle et technologique de développer des organismes transgéniques. Une majorité de cas d'applications concernera probablement la responsabilité civile puisqu'il s'agira de transactions commerciales avec des entreprises privées. Cependant, la question de la responsabilité étatique qui n'a reçu que peu d'attention jusqu'à présent devra être considérée en détail. En effet, il est à craindre que des situations similaires au problème de la 'biopiraterie' ne se développent dans le futur. Dans le cas où, par exemple, une aide alimentaire transgénique cause un dommage environnemental, sanitaire ou socio-économique, l'absence de règles strictes de responsabilité étatique risque soit de forcer les pays du Sud à se retourner contre l'Etat d'origine au for du défendeur soit à abandonner toute prétention en réparation.

\section{CONCLUSION}

Ce chapitre a analysé les différentes caractéristiques du dommage potentiel lié à l'introduction d'organismes transgéniques dans l'environnement. L'existence de trois types de dommages constitue une spécificité de la biotechnologie. Elle confirme que les questions liées à la responsabilité dans le domaine de la biotechnologie sont spécifiques à ce domaine et nécessitent des solutions adaptées. Le développement d'un régime de responsabilité spécifique à la biotechnologie est donc nécessaire. Ceci est vrai tant au niveau national qu'au niveau international. Dans une large mesure, les mêmes problèmes se posent aux deux niveaux hormis les questions posées par la responsabilité étatique qui ne se posent qu'au niveau du droit international.

Le développement d'un régime de responsabilité dans le cadre du Protocole de Cartagena au cours des quatre ans à venir va permettre aux Etats d'analyser plus en détail les questions soulevées dans ce chapitre et de donner des réponses appropriées au niveau international et national. Une grande majorité de pays devra poursuivre les deux processus en parallèle étant donné que la plupart des pays n’ont pas encore développés de règles de responsabilité pour la biotechnologie.

s5 Voir généralement Usha Ramanathan, A Critical Analysis of the Laws Relating to Compensation for Personal Injury (PhD thesis, Delhi University, 2002). 